\title{
Comparación del aprendizaje en internet con la clase convencional en estudiantes de medicina, en Argentina.
}

\author{
Comparative study between learning on line and traditional lectures \\ for medical students in Argentina.
}

Bogdan Mihai Popescu, Viviana Navarro.

Facultad de Medicina de la Universidad Nacional del Nordeste. Argentina

Se considera que los estudiantes aprenden igual o mejor en la web que en clases tradicionales.

Sin embargo no abundan ensayos randomizados que brinden evidencia al respecto, especialmente en Argentina.

Objetivo: Comparar el aprendizaje sobre tratamiento de la diabetes(TD)entre dos grupos de estudiantes avanzados de medicina, uno con clase tradicional y otro utilizando Internet.

Materiales y métodos: 120 estudiantes de una Cátedra de Medicina de la Universidad Nacional del Nordeste, Argentina, contestaron un cuestionario con 20 preguntas sobre TD. Tres meses después, fueron randomizados en $\mathbf{2}$ grupos para participar en una clase 0 en un foro de Internet sobre (TD). Los estudiantes no conocían el objetivo del estudio. Todos utilizaron la misma bibliografía. La clase tuvo una duración de $\mathbf{2}$ horas, conectándose al mismo tiempo el grupo de Internet a un foro habilitado durante 48 hs. 70 horas después de finalizada la actividad realizaron el mismo cuestionario inicial sobre TD.

Resultados: Fueron incluidos 107 estudiantes (13 fueron excluidos por no completar alguno de los 2 cuestionarios). La puntuación promedio del grupo clase, antes, fue de $8.30 \pm 2.48$ y después de $9.76 \pm$
Correspondencia:

Bogdan Mihai Popescu,

Moreno 1240.3400 Corrientes. Argentina

Telefax:54-3783-426306

e-mail: bpopescu@med.unne.edu.ar
2.17. El grupo Internet obtuvo una puntuación antes de $8.39 \pm 2.14$ y después de $9.76 \pm 2.8$, $p>0.10$ en la comparación de los grupos. Conclusión: Se demuestra que el aprendizaje de un tema de terapéutica para estudiantes de medicina argentinos fue igualmente efectivo por Internet que con una clase convencional.

Palabras clave: Estudio comparativo-Educación de pregrado en Medicina/ Métodos-Instrucción por computación

It is not known if medical students can learn better or not with online methods than with traditional lectures. There are not many randomized studies that substantiate this belief, especially in Latin American settings.

Purpose: To compare two different methods of learning diabetes' treatments (DT) in two groups of advanced medical students. One group used an online forum, the other group a traditional lecture. Method: We conducted surveys among 120 medical students of the Northeastern National University of Argentina. The first survey was a questionnaire of 20 items about DT. The students were then randomized to two groups: one assigned to traditional lecture, and another assigned to an online Forum about DT. The students were not aware of the study's purpose. The same guidelines were provided to both groups. Duration of the lecture was 2 hours. The group working online was connected at the same time to the Web forum for $\mathbf{4 8 h}$. 70 hours after the lecture and the on line exposure all students repeated the initial questionnaire. 
Results: 107 students were included

( 13 were excluded because they didn't complete one of the questionnaires). The average performance of the lecture's group was $8.30+/-2.48$ points before, and $9.76+/-2.17$ after the educational intervention. In the Web program group it was $8.39+/-2.14$ points initially, and $9.76+/-2.8$ after. The $p$ was $>0.10$ comparing the lectures and the internet groups.

Conclusion: Learning a therapeutic subject by Argentinean students was equally as effective with Web based learning as with traditional lectures.

Key Words: Comparative study, Medical Undergraduate Education, Internet-Lectures, Web based learning-online learning

\section{INTRODUCCIÓN}

Si bien el uso de computadoras en Medicina viene siendo utilizado desde hace muchos años, sólo recientemente se ha generalizado su uso en la enseñanza por las innovaciones que han permitido el fácil acceso a Internet y la posibilidad de usar sitios y paquetes de Multimedia destinados a la enseñanza de la Medicina. Este uso ha producido una verdadera revolución electrónica ${ }^{1}$ y se ha aplicado el aprendizaje en Internet en Medicina para el pre y postgrado, e inclusive se ha usado como soporte para el desarrollo del curriculum en varias faculta$\operatorname{des}^{2-3}$. Se ha considerado que los alumnos aprenden igual o mejor en la red que en las clases presenciales tradicionales sin embargo en la literatura no abundan ensayos randomizados que brinden un fundamento basado en la evidencia de esta aseveración ${ }^{4-5-6}$, menos aún en países Latinoamericanos, donde el acceso a Internet es más difícil no sólo por problemas económicos que dificultan su disponibilidad sino también porque el idioma inglés constituye una barrera para acceder a la información disponible en la red.

El objetivo de este trabajo ha sido comparar la adquisición de conocimientos sobre el tratamiento de la diabetes entre dos grupos de estudiantes de medicina avanzados, uno participando en una clase convencional y otro utilizando Internet.

\section{MATERIAL Y MÉTODOS}

A los 187 estudiantes de la IV Cátedra de Medicina de la Universidad Nacional del Nordeste se les realizó una encuesta auto administrada con 6 preguntas cerradas acerca de sus conocimientos sobre manejo de computadoras, Internet e inglés. Luego completaron un cuestionario con cuatro problemas clínicos sobre tratamiento de diabetes con 5 preguntas cada uno, cada pregunta tenía un valor de un punto y la puntuación máxima que se podía obtener era de 20 puntos.

Tres meses más tarde se invitó a dichos estudiantes a participar voluntariamente en un taller sobre Tratamiento de la Diabetes en el cual participaron 120 estudiantes los cuales fueron randomizados al azar para participar en una clase tradicional presencial y/o en un foro de Internet sobre el tema mencionado. Se randomizaron en forma aleatoria por asignación de números constituyéndose 2 grupos de 60 estudiantes cada uno, uno para la clase tradicional y uno para el foro de Internet. Los alumnos no conocían los objetivos del estudio. A cada grupo se le asignó un tutor, Jefe de trabajos prácticos de la mencionada cátedra.

A ambos grupos se les proporcionó la misma bibliografía, a los de Internet a través de la red y al grupo clase en un cuadernillo. La clase presencial fue interactiva y duró 2 horas; al mismo tiempo comenzó la conexión del grupo asignado a Internet a un sitio web con un Foro habilitado a tal efecto durante 48 horas con participación de sus tutores, pudiendo los estudiantes comunicarse con ellos también por vía e-mail.

Los alumnos de ambos grupos fueron citados nuevamente 70 horas después de finalizada la clase siendo sometidos al mismo cuestionario que tres meses antes sobre TD.

Análisis Estadístico: El cálculo del tamaño de la muestra para Test de la $t$ fue calculada con un alfa de 0.05 y un beta 0.10 requiriéndose 50 individuos para cada grupo. Realizándose el análisis de los grupos con test de la $t$ apareada o por parejas. Se calcularon además medidas de tendencia central y dispersión e intervalo de confianza.

\section{RESULTADOS}

Los datos fueron analizados excluyéndose del grupo de 120 estudiantes a 13, debido a que no realizaron alguno de los cuestionarios, por lo que fueron incluidos para el presente estudio 107 alumnos.

Los resultados de la encuesta inicial sobre el perfil de los 107 alumnos se muestran en la tabla 1.

El rango de puntación y la puntación promedio del grupo de clase y del grupo de Internet antes y 
Tabla 1 : Evolución de los puntajes alcanzados por los estudiantes antes y después de la intervención

\begin{tabular}{|c|c|c|c|c|c|}
\hline $\begin{array}{l}\text { Rango de puntajes } \\
\text { ANTES de } \\
\text { la intervención }\end{array}$ & $\begin{array}{l}\text { Rango de puntajes } \\
\text { DESPUÉS de } \\
\text { la intervención }\end{array}$ & $\begin{array}{l}\text { Promedio de los } \\
\text { puntajes ANTES }\end{array}$ & $\begin{array}{l}\text { Promedio } \\
\text { de los puntajes } \\
\text { DESPUÉS }\end{array}$ & $\begin{array}{l}\text { Valor de las } \\
\text { diferencias en un } \\
\text { mismo grupo antes } \\
\text { y después de } \\
\text { la intervención }\end{array}$ & $\begin{array}{l}\text { Prueba de la t } \\
\text { que compara } \\
\text { las diferencias } \\
\text { entre ambos } \\
\text { grupos }\end{array}$ \\
\hline
\end{tabular}

\begin{tabular}{|c|c|c|c|c|c|c|}
\hline \multicolumn{7}{|l|}{ Grupo } \\
\hline Clase & $4-13$ & $5-16$ & $8.3 \pm 2.4$ & $9.7 \pm 2.1$ & $\begin{array}{l}\text { IC } 90 \% \\
p<0.001\end{array}$ & $\mathrm{~T}=0$ * \\
\hline $\begin{array}{l}\text { Grupo } \\
\text { Internet }\end{array}$ & $5-13$ & $5-17$ & $8.3 \pm 2.1$ & $9.7 \pm 2.8$ & $\begin{array}{l}\text { IC 90\% } \\
p<0.01\end{array}$ & $\mathrm{~T}=0$ * \\
\hline
\end{tabular}

* No existe diferencia estadísticamente significativa entre los promedios alcanzados por cada grupo al final

después de la intervención se puede observar en la tabla 1.

Al comparar el promedio de las diferencias aplicando la prueba de la $t, p$ es $<0.001$ a favor del grupo Internet. Esta diferencia se explica si observamos que la Desviación Standard es mayor en este grupo.

Al aplicar el Test de la t para evaluar si existe diferencia estadísticamente significativa entre los promedios alcanzados por cada grupo al final, el valor fue de $\mathrm{t}=0$ por lo cual se establece que tal diferencia no existe.

\section{DISCUSIÓN}

Los resultados de nuestra evaluación posterior a la enseñanza sobre un tema de terapéutica clínica por Internet, demuestran que la misma fue igualmente efectiva para la adquisición de conocimientos que una clase convencional.

Este hallazgo resulta particularmente útil dado que si bien el uso de Internet se ha aplicado cada vez en forma más frecuente para la enseñanza de la Medicina, existen en la literatura escasos ensayos randomizados que demuestren superioridad o igualdad de eficacia pedagógica del uso de Internet con respecto a las clases tradicionales.

Es interesante señalar que ambos grupos de estudiantes que participaron del estudio eran de perfil similar, con pobres antecedentes de navegar en la web (solamente del 44 al $50.8 \%$ se había conectado al medline alguna vez) y pobre conocimiento del inglés (el 37,5\% no podía leer un artículo en inglés) que es una de las barreras para el uso completo de los recursos disponibles en la red, perfil que puede ser similar a la población estudiantil de otras Facultades argentinas e inclusive latinoamericanas, a pesar de lo cual los estudiantes tuvieron en Internet el mismo rendimiento que en la clase convencional. (Figura 1)

Estas conclusiones permitirían alentar el uso de Internet en nuestras Facultades considerando que esta nueva herramienta educacional ofrece diversas ventajas $^{7}$ tales como: permitir un estudio más independiente y activo, acceder con facilidad a textos o revistas médicas online, llegar con materiales visuales a un mayor número de alumnos, favorecer una mejor interacción con los docentes y sus pares vía los foros, facilitar la acción de los docentes que

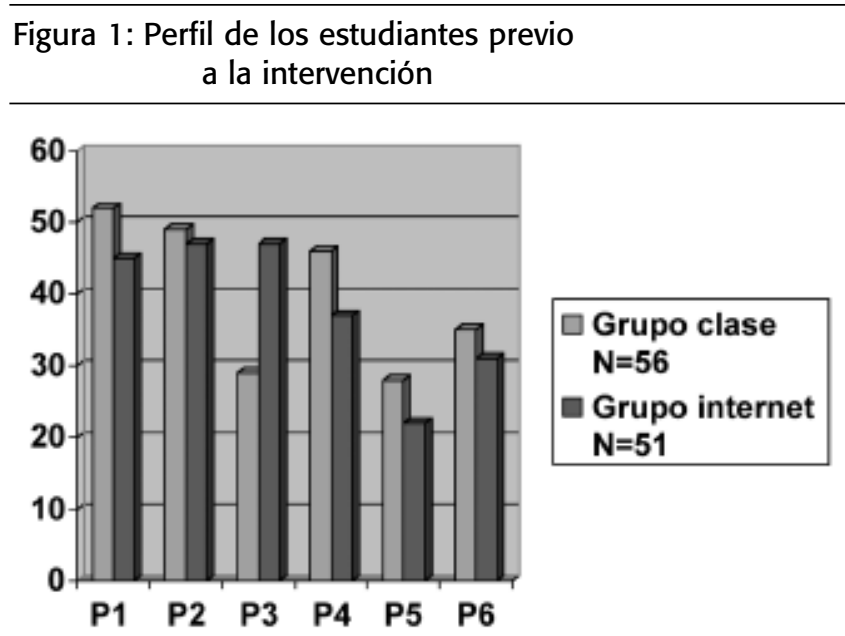

Pregunta(P).P1: Usó computadora alguna vez?. P2: Tiene acceso a una computadora?. P3: Tiene computadora propia?. P4 : Navegó en Internet alguna vez?. P5: Usó Medline alguna vez?. P6: Puede leer artículos en inglés? 
pueden construir una vez y utilizar el material indefinidamente, facilitar el aprendizaje de los estudiantes con menor tiempo de concurrencia activa a la Facultad lo cual sería una ventaja para los que viven lejos o trabajan y para Facultades con pocos espacios disponibles. Sin por ello desconocer las dificultades que ofrece: tener equipamiento adecuado, para que los alumnos puedan hacer un buen uso del recurso y no se sientan frustrados por dificultades técnicas, la despersonalización que puede traer su uso generalizado. Hay inclusive interrogantes no aclarados como cual será la motivación y el compromiso cognoscitivo de los que utilicen este tipo de aprendizaje $^{8}$. Por otra parte el uso de Internet permite el acceso a la información pero esto es solo uno de los elementos del juicio clínico?.

Como conclusión consideramos que el presente estudio brinda evidencia que apoya el uso de Internet para la enseñanza de nuestros estudiantes lo cual les permitiría acceder a nuevas modalidades de aprendizaje ${ }^{10}$.

\section{BIBLIOGRAFÍA}

1. Chodorow S., Educators must take the electronic revolution seriously. Ac Med.1996;71:221-226.
2. Roberts C, Lawson M, Newble D, Self A. Managing the learning environment in undergraduate medical education: the Sheffield approach. Med Teach. 2003 May;25 (3):282-6.

3. Ellaway R, Dewhurst D, Cumming A. Managing and supporting medical education with a virtual learning environment: the Edinburgh Electronic Medical Curriculum. Med Teach. 2003 Jul;25(4): 372-80.

4. Lipman A.J., Sade R.M., Gloltzbach A.L., Lan-caster C.J., Marshall M.F., The Incremental Value of Internet-based Instruction as an Adjunct to Classroom Instruction. Ac Med.2001; 76:1060-1064

5. Maio M. de, et al. ,Experience with the first Internet-based course at the Faculty of Medicine, University of Sao Paulo. Rev. Hosp. Clín. Fac. Med. S. Paulo 56 (3):69-74,2001

6. Leasure AR, Davis L, Thievon SL, Comparison of student outcomes and preferences in a traditional vs. World Wide Webbased baccalaureate nursing research course. J Nurs Educ. 2000 Apr;39(4): 149-54.

7. McKimm J, Jollie C., Cantillon P., Web based learning, BMJ 2003; 326:870-873

8. Clark D. Psychological myths in e-learning. Med. Taech.2002; 24:598-604

9. Klass D.J., Will e-learning will improve clinical judgment?. BMJ 2004;328;1147-1148

10. Tao L., Stein M., Medical Education and the Internet: This Changes Everything. JAMA 2001;285:809 